\title{
Von der Universalität der Liebe und den ewigen Unwahrheiten der Geschichte. Antikerezeption in den Gedichten von Aleksej Cvetkov
}

\author{
Marion Rutz \\ Universität Passau, Lehrstuhl für Slavische Literaturen und Kulturen, \\ D-94030 Passau, Deitschland; marion.rutz@uni-passau.de
}

For citation: Marion Rutz. Von der Universalität der Liebe und den ewigen Unwahrheiten der Geschichte. Antikerezeption in den Gedichten von Alexej Cvetkov. Philologia Classica 2018, 13(1), 151-164. https://doi. org/10.21638/11701/spbu20.2018.112

Going through what has been written about the reception of antiquity in Russian literature, one detects quite huge unexplored areas. The second half of the 20th century as well as contemporary (i.e. post-Soviet) literature are - with very few exceptions - beyond the scope of research. The article draws attention to the well-known underground and then émigré poet Aleksei Tsvetkov $\left.{ }^{\star}{ }^{\star} 1947\right)$, who after 2001/2006 saw an exceptional comeback. References to Greek and Roman literature and history are frequent in his oeuvre. In about two dozens of texts these motifs are dominant, three of these poems are analyzed in the article (latyn', domashnee chtenie, rasskaz ochevidtsa). One of the most important aspects in Tsvetkov's creative reception of the classics is that his poems are not only occupied with conceptualizing our connection to ancient culture and demonstrating the vitality of at least some literary topics (love, separation) which are able to bridge the temporal and spatial distance. They also deal with controversial aspects of the Greek and Roman legacy, such as the Trojan War, the Thermopylae myth or Alexander's imperial aspirations. In rasskaz ochevidtsa, Tsvetkov links the fate of Callisthenes, historiographer in the service of Alexander the Great, to the experiences of Stalinism and - referring to Polish literature - the Warsaw uprising in 1944.

Keywords: Aleksei Tsvekov, ancient legacy in modern Russian poetry.

Texte entstehen aus vorhandenen Texten. Auch wenn man nicht mit poststrukturalistischer Radikalität den Autor töten und die Leerstelle durch Intertextualität ersetzen möchte, bleibt die Tatsache, dass Texte immer vor dem Hintergrund von und in der Auseinandersetzung mit früheren Texten geschrieben werden. Das fortwährende Aktualisieren und Variieren ist ein wichtiger Mechanismus der literarischen Produktion und sorgt für das Weiterleben des literarischen Erbes. Eine der ältesten Literaturschichten, die von Generation zu Generation weitergereicht werden, sind im europäisch geprägten Kulturkreis die Texte der griechischen und lateinischen Antike. Das gilt auch für Russland, obgleich die Wahrnehmung als Teil des Eigenen hier nicht immer selbstverständlich war. Heute verfügen die Reception Studies über einen recht lebendigen russischen Zweig. Was das thematische Reservoir angeht, fallen im internationalen Vergleich (Riedel 2000, Hardwick 2003) allerdings zwei Unterschiede ins Auge: Monographien und Sammelbände enden um 1900 (Knabe 1996, Wes 1992) oder bei den Klassikern des „Silbernen Zeitalters“ (Mal'chukova, Dekht'arenok, Nilova, 2011; Frajlich 2007). Der jüngste überhaupt berücksichtigte Autor ist Josif Brodskij (1940-1996) (Uspenskaja 2008, 274-281; in Tacho-Godi 2015 zwei Beiträge: 515-540, 567-574). In den Gesamtdarstellungen ist damit nicht nur

(C) St. Petersburg State University, 2018 
die jüngere Gegenwart, sondern mit Ausnahme des emigrierten Nobelpreisträgers Brodskij / Brodsky auch die zweite Hälfte des 20. Jahrhunderts terra incognita. Gedichtanthologien reichen ebenfalls nur zu den 1930-1940er Jahren (Golodnikov 2012; Gvozdeva 2014; vgl. Seidensticker, Habermehl 1996). Eine Ausnahme ist Martirosova Torlones Monographie Russia and the Classics (2009), die die Antikerezeption bis in die Gegenwart verfolgen möchte. Sie springt ebenfalls von Mandel'štam zu Brodskij, wagt dann im Schlusskapitel einen kurzen Ausblick auf das Jetzt und trägt Namen zusammen (197f.). ${ }^{1}$

Es mag vereinzelte Beiträge zur Antikerezeption bei anderen Autorinnen und Autoren der zweiten Hälfte des 20. und des 21. Jahrhunderts geben, allerdings sind diese wenig sichtbar. Das gilt auch für die russischsprachige, nicht als Buch publizierte Dissertation von Cypilëva zur Rezeption der Antike bei den Dichtern Arsenij Tarkovskii (1907-1989), Aleksandr Kushner $\left({ }^{*} 1936\right)$ und Sergej Stratanovskij $\left({ }^{\star} 1944\right)$ aus dem Jahr 2016. Was hier auffällt und insgesamt charakteristisch ist, ist die stets positive Wertung des antiken Erbes. Das Autoreferat von Cypilëva $(2016,3)$ beginnt mit dem Gedanken, dass das Interesse an der Antike im an Umbrüchen reichen 20. Jahrhundert durch die Suche nach Halt und den Versuch, die traditionellen Werte zu bewahren, motiviert sei. Vor dem Hintergrund der internationalen Forschung verwundert die Selbstverständlichkeit, mit der die dieses positive Führungszeugnis ausgestellt wird. Das griechisch-römische Ideal wurde im italienischen Faschismus und deutschen Nationalsozialismus propagandistisch ausgeschlachtet (Hardwick 2003, 43-50), und zumindest in der deutschsprachigen literarischen Rezeption finden sich nach Auschwitz und Stalingrad Brüche (vgl. Riedel 2000, 321-327). Gab es eine ähnliche Instrumentalisierung nicht auch in der UdSSR, und gibt es vergleichbare kritische Gegenreaktionen (ein Beispiel wäre Brodskijs dystopischer Zyklus Post aetatem nostram, siehe Martirosova Torlone 2009, 174f.; Chagi 2010)? Vielleicht lässt sich auch in der russischen Literatur finden, was Riedel $(2000,7)$ für die deutsche einfordert: statt „einer weitgehend auf Verehrung und Weiterführung hin angelegten Antikerezeption" eine stärkere Berücksichtigung von Momenten, die „eine problematisierende, entheroisierende und entidealisierende Sicht auf tradierte Motive“ befördern.

In vorliegendem Beitrag soll das Werk zumindest eines Gegenwartsdichters vorgestellt und nach neuen und v. a. kritischen Perspektiven gesucht werden. Dass die Wahl auf keinen der von Martirosova Torlone genannten Namen und auch nicht auf die für ihre Experimente mit antiker Metrik bekannten Sergej Zavjalov ( $\left.{ }^{\star} 1958\right)$ und Maksim Amelin $\left({ }^{\star} 1970\right)$ fiel, sondern auf Aleksej P. Cvetkov, ist im Ursprung einem Zufallsfund geschuldet: dem in der Literaturzeitschrift Znamja [Banner] gelesenen Gedicht latyn' [Latein] (Cvetkov 2007a, 98). Diese Entscheidung lässt sich allerdings durch Sachargumente absichern: Aleksej Cvetkov ist ein in der Dichterszene und unter Experten bekannter und renommierter Dichter. 1947 in der Ukraine geboren, wurde er nach der Übersiedlung von Odessa nach Moskau und einem Wechsel vom Studium der Chemie zu Geschichte und Journalistik in offiziellen und inoffiziellen literarischen Kreisen der 1960er Jahre heimisch. Im Jahr 1975 emigrierte er unter ungeklärten Umständen in die USA. Er promovierte 1981 an der University of Michigan mit einer slavistischen Arbeit zu Andrej Platonov und begann nach einem Intermezzo als Dozent für Radiostationen zu arbeiten, die ein alternatives russischsprachiges Programm in den Osten ausstrahlten: zuerst

${ }^{1}$ Andrej Poljakov, Ol'ga Grebennikova, Demjan Kudrjavcev, Konstantin Uvarov, Polina Barskova, Grigorij Starikovskij. 
Voice of America (Washington D.C., 1985-1989), danach Radio Liberty (München, nach 1995 Prag). ${ }^{2}$ Heute lebt der Dichter in den USA.

Aleksej Cvetkov gehört zu den Autoren der sog. dritten russischen Emigration. Er konnte recht bald nach seiner Ankunft in den USA literarisch Fuß fassen. Der legendäre Emigrationsverlag Ardis publizierte 1978, 1981, 1985 drei Gedichtbände, und die anglophone Russistik wurde auf die neue Stimme aufmerksam (Levitsky 1985; Smith 1986). Nach diesem geglückten Start verstummte der Dichter unerwartet für über ein Jahrzehnt. Erst 2001 publizierte der Petersburger Verlag Puškinskij fond den Band Divno molvit' [Wundersam zu sagen], der neben den in den USA erschienenen Texten auch eine Auswahl neuer Gedichte enthält, die das Schweigen beendeten. Das eigentliche Comeback erfolgte jedoch erst 2006 mit dem Band Šekspir otdychaet [Shakespeare ruht aus], auf den weitere Publikationen in renommierten Verlagen folgten. Der sechzigjährige Cvetkov stieg zu einem der wichtigsten aktuellen Dichter auf (vgl. Skvorcov 2008, 189). Seit 2012 erscheinen neue Bücher im New Yorker Verlag Ailuros Publishing, u. a. 2015 die zweibändige Gesamtausgabe Vse èto, ili Ėto vse [All das, oder Das ist alles].

Wie das Aneinanderreihen von Gedicht an Gedicht in der Gesamtausgabe zeigt, ${ }^{3}$ spielen übergeordnete kompositorische Einheiten bei Cvetkov in der Regel eine geringe Rolle. Auch die Gedichte mit Antikebezug verdichten sich nicht zu Zyklen oder thematischen Büchern. Dies, wie auch die Tatsache, dass die Buchtitel nirgends auf die Antike Bezug nehmen, ${ }^{4}$ mag dazu beigetragen haben, dass sich bislang nur ein Literaturwissenschaftler mit Cvetkovs Antikerezeption beschäftigt hat. Die rudimentären Publikationen von Artem Skvorcov vermitteln allerdings ein nur sehr oberflächliches Bild. ${ }^{5}$ Zentrale Themen seien die Fremdheit der antiken Welt und die große, unüberwindbare Distanz zum Jetzt; die Antike stünde weit über den moralischen Maßstäben der Gegenwart (Skvorcov 2004, Skvorcov 2011, 24).

Meine Fragen gehen in eine ähnliche Richtung, jedoch unterscheiden sich die Erkenntnisziele, die Antworten sowie auch die (aktuellere) Textauswahl. Es wird zum einen untersucht, welche Aspekte der Antike Cvetkovs Gedichte überhaupt als relevant für das eigene Schreiben markieren und wie die trennende Distanz in den Texten modelliert und überwunden wird. Zum anderen werden kritische Perspektiven gezeigt, die über das stereotype Idealisieren der Griechen und Römer hinausgehen. Hierbei spielt vor allem historiographische Sujets eine zentrale Rolle.

\section{Lassen sich Jahrtausende überbrücken: latyn'}

Die Liste der Gedichte, die antike Motive enthalten, ist lang (es gibt auch einen Fragment gebliebenen historischen Roman, Prosto golos [Nur Stimme]: Cvetkov 2002, 5-209). Sortiert man diejenigen aus, die nur punktuell Realien, Namen, mythologische Metaphern

2 Die biographischen Angaben nach Gresta $(2007,106 f)$.

3 Die Texte der früheren Ausgaben sind - mit Ausnahme der 1970-1978 geschriebenen Gedichte in $\mathrm{Bd} .2$ - ohne veränderte Sortierung zusammengeführt. Allerdings fehlen an vielen Stellen einzelne Gedichte, als ob diese aussortiert worden wären. Textgrundlage für vorliegenden Beitrag sind daher die Erstausgaben der Bücher.

4 Allein das Foto einer ionischen Säule auf dem Cover von rovnyj veter (Cvetkov 2008) ist ein Hinweis.

${ }^{5}$ Drei Seiten über die Antikerezeption bei Cvetkov und Brodskij (Skvorcov 2004), die Zusammenfassung der Habilitationsschrift (Skvorcov 2011, 24), ein literaturkritischer Artikel (Skvorcov 2008, 191f.), ein Interview (Cvetkov, Skvorcov 2007, 245-247). 
oder lateinische Zitate verwenden, sowie die, in denen die Bezüge zwar häufiger sind, aber zur Illustration eines anderen Themas dienen, bleiben gut zwanzig Texte übrig. Diese eigentlichen Antikegedichte lassen sich grob einteilen in solche, in denen literarische Sujets im Zentrum stehen, und solche mit historiographischer Basis. Was die literarischen Stoffe angeht, geht es auffällig oft um Liebe, Trennung und/oder Tod. Fünf Gedichte schöpfen aus der Ilias, zwei beleuchten die Beziehung zwischen Odysseus und Penelope. ${ }^{6}$ In der römischen Literatur liegt ein besonderer Fokus auf der Dido-Episode aus Vergils Aeneis sowie der Plejade der Liebeslyriker. Diesen Schwerpunkt setzt v. a. das eingangs erwähnte Gedicht latyn' [latein] (Textgrundlage ist Cvetkov 2007b, 71). Wie der Titel nahelegt, beschäftigt sich das Gedicht grundsätzlich mit der Relevanz der „toten“ Sprache und Literatur für die Gegenwart - darum macht es Sinn, hier mit der Suche nach der Bedeutung der Antike bei Cvetkov zu beginnen.

Vor dem Einstieg in die Analyse ist auf zwei Markenzeichen Cvetkovs hinzuweisen, die seit dem Band Sostojanie sna [Schlafzustand] (1981) Konstanten seiner Poetik sind. In den Gedichten wird auf Interpunktionszeichen und jegliche Art von Großschreibung verzichtet (auch bei Namen: propercij, vergilij, dido, ...). Beide Normverstöße bewirken einen starken Verfremdungseffekt. Gedichte, die sich ansonsten im Rahmen vertrauter Konventionen halten - in latyn': Strophen à 4 Verse, weiblich-männlich alternierende Kreuzreime, 5-hebiger Jambus - wirken innovativ und modern. Die der Leserin bzw. dem Leser abverlangte syntaktische Rekonstruktion fordert Konzentration, wird jedoch dadurch erleichtert, dass Syntagmen in der Regel am Versende enden und die Syntax einfach ist. Die fehlende Interpunktion ermöglicht eine freiere, assoziative Anordnung von Inhalten sowie semantischen Unschärfen.

Cvetkovs Gedicht latyn' handelt von der Kontaktaufnahme mit der entfernten Welt der Antike und prägt in Strophe 2 (V.5-8) Metaphern, die diese Distanz ausdeuten:

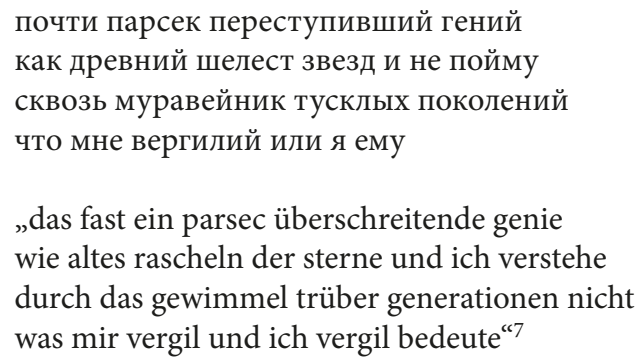

Bei dem Parsec (parallaktische Sekunde), das das „Genie“ (Vergil) überbrückt, handelt es sich um eine astronomische Längeneinheit, die 3,26 Lichtjahren entspricht. Dass das Wort parsek ausgewählt wurde, und nicht das vertrautere svetovoj god, hat poetische Gründe (Silbenzahl und die Alliteration počti parsek perestupivšij), denn gerade ,Lichtjahr' verkörpert die Verschmelzung von Raum und Zeit, die für das Gedicht wichtig ist. Aufgrund der Dimension der Entfernungen im Weltall legt das Licht auch eine Strecke in der Zeit zurück. Das Licht der Sterne ist nicht nur Jahre, sondern Jahrtausende bis zur

6 Ilias: „Seryj koršun planiroval v lesu...“(Cvetkov 1978, 25f.), Proščanie Gektora s Andromachoj (60f.), Plač Andromachi (62f.), Sentimental'naja ballada (Cvetkov 1981, 82f.), achejskaja pesnja (Cvetkov 2010, 130f.). Odyssee: „s kolosnikov provorny provoda..." (Cvetkov 2006, 38), tkačicha (Cvetkov 2008, 21f.).

7 Alle Übersetzungen aus dem Russischen stammen von der Verf. 
Erde unterwegs, und beim Blick in den Nachthimmel sieht man in der Gegenwart die Vergangenheit. Im Gedicht wird diese kosmische Metaphorik in V.6. durch den Vergleich „wie altes Rascheln der Sterne“ fortgeführt. Auch Radiowellen sind lange unterwegs, šelest ('Rascheln, Rauschen') verbindet die Assoziation von ins All gerichteten Radioteleskopen mit dem Geräusch der in V. 1f. umgeblätterten Buchseiten.

Der Transport literarischer Momentaufnahmen aus der Vergangenheit vollzieht sich allerdings nicht störungsfrei in einem weitgehend leeren Kosmos, sondern "durch ein gewimmel trüber generationen“ (V.3). Muravejnik, wörtlich 'Ameisenhaufen', bezeichnet die Vermittler der ursprünglichen Signale: die Generationen von Lesern - vielleicht sogar von anonymen (daher die Attribuierung als 'trübe Generationen') Bibliothekaren, Schreibern und Gelehrten, deren unermüdlicher Arbeit die Überlieferung der antiken Literatur $\mathrm{zu}$ verdanken ist. Während die Texte die Jahrhunderte überwunden haben und in die Gegenwart gelangt sind, haben Sie dennoch einen Teil Ihrer Botschaft verloren. Sie sind zu einem (leisen, unverständlichen) „Rauschen“ (V.6) geworden und bieten kaum noch Berührungspunkte mit dem Heute, vgl. am Ende der Strophe: „и не пойму <...> что мне вергилий или я ему“ [und ich verstehe nicht was mir vergil und ich vergil bedeute]. Hier greift Skvorcovs These von der bei Cvetkov unüberbrückbaren Distanz (mit diesem Vers als Beleg: Skvorcov 2008, 191), und in der Tat sind viele antike Texte heute kaum noch oder nur für Fachleute verständlich und lesenswert. Allerdings ist Strophe 2 nicht der Endpunkt der Argumentation. Aus den folgenden Strophen ergibt sich ein optimistischeres Fazit, denn zumindest ein Teil der ursprünglichen Botschaften erreicht das im Gedicht sprechende „Ich“.

Die Auswahl der antiken Autoren und Texte, auf die angespielt wird, suggeriert, dass vor allem Texten über Liebe und Trennung die raum-zeitliche Entfernung überwinden. Der Katalog intertextueller Referenzen beginnt in V.3 mit einem Verweis auf „die sechste Elegie von Properz" (aus Buch 1). Das Nennen eben dieser Elegie könnte mit subjektiven Vorlieben des Autors zusammenhängen, der dieses Gedicht nachgedichtet hat (Cvetkov 2017), es passt jedoch auch zum in latyn dominanten Themenspektrum ,Liebe und Abschied‘. Properz' Sprecher („lyrisches Ich“) erklärt, dass ihn die Liebe zu Cynthia in Rom festhalte, und malt den zu erwartenden Abschiedsschmerz der Geliebten aus. Von Properz springt Cvetkovs Gedicht zu Vergil. Aufgerufen wird in V. 4 und erneut in V. 9f. Didos Suizid am Ende von Buch 4 der Aeneis. ${ }^{8}$ Dass aus der Aeneis das Liebesthema herausgegriffen wird, ist weniger selbstverständlich als im Fall von Properz. Das Gedicht klammert die an sich wichtigere, aber weniger unmittelbar verständliche politisch-propagandistische Bedeutungsdimension aus. Der dritte intertextuelle Verweis bezieht sich wieder auf einen typischen Text über die Liebe; V. 11 erwähnt Delia, die Geliebte aus den Elegien des Tibull. Wenn in latyn' Delia den Dichter ins Elysium begleitet, verschmelzen dabei zwei Stellen (Tibull, Buch 1, Elegie 1, V.59-66 und Buch 1, Elegie 3, V.58). In V.15f. komplettiert Catull mit Lesbia das Pantheon. Der gemeinsame Nenner, Abschied / Tod' ist hier mit dem toten Sperling präsent, über den Lesbia in Gedicht 3 Tränen vergießt.

Verse über Liebe und Trennung sprechen Cvetkovs „Ich“ an, gierig blättert es bzw. er abends durch die Gedichte (V. 1: „Vzachleb stichi listaja“). Die immateriellen Texte rufen sogar körperliche Reaktionen - ein Stechen in der Brust - hervor (V.13f. „, grud' kol'nula / zarja želannych glaz rumjanec skul“). Die identische emotionale Erfahrungs-

8 Vergil wird auch in V. 8 erwähnt; Cvetkov nennt ihn im Interview seinen zweitliebsten Autor — nach Shakespeare (Cvetkov, Skvorcov 2007, 246). 
welt hebt den linearen Lauf der Zeit auf, was sich an den temporalen Indikatoren in Str. 4 erkennen lässt: Catulls Sperling wird zum einem mit dem Adjektiv „ewig“ attribuiert (večnyj vorobej) und scheint andererseits erst "gestern“ (včera) entschlafen zu sein. Cvetkov holt aber nicht nur die antiken Texte ins Jetzt, sondern lässt seinen Sprecher in die Welt der Vergangenheit eintreten. Der Übergang erfolgt über den Schlaf / Traum (russ. son meint beides). In Strophe 1 verfällt der Sprecher zuerst in einen leichten Schlummer, reale Geräusche und konturierte Erinnerungen an gelesene Texte sind noch präsent. Die Tiefschlafphase setzt zwischen Str. 4 und 5 ein, markiert durch die Anadiplose usnul [entschlummerte] in V. 16 und V.17. Der Übergang vollzieht sich durch eine Art Schleuse aus morphologisch verwandten Antonymen: „usnul i ja prosnut'sja v drevnem rime“ (V.17) [auch ich schlief ein um im alten rom zu erwachen].

Die letzten Verse des Textes formulieren ein Fazit und knüpfen an den Gedichttitel an. Dem „toten“ Latein wird eine paradoxe Lebendigkeit und Wirkmächtigkeit zugeschrieben:

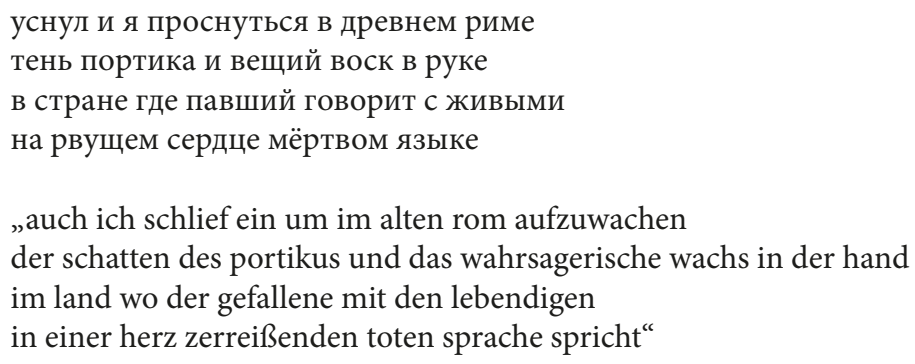

Dass im geträumten Rom Latein gesprochen, engt diese Wirkung der lateinischen Originale jedoch genaugenommen auf sprachlich kompetente Leserinnen und Leser ein, zu denen der Autor sich selbst zählt (Cvetkov, Skvorcov 2007, 245f.). Denen, die sich mit Übersetzungen begnügen müssen, stellt latyn' zumindest ein verlockendes Ideal vor.

\section{Was hält der kritischen Reflexion stand: domašnee čtenie}

Die in latyn' katalogisierten Liebesklassiker tauchen sporadisch in weiteren Gedichten auf (z. B. smert didony [der tod der dido]; Cvetkov 2012, 129), für den Gesamtzusammenhang wichtig ist besonders „čut' gormonal'naja nakryla grust'... “ [„eine leicht hormonelle sehnsucht hat mich erwischt..."]. Hier erinnert sich der Sprecher daran, wie ein pubertierendes jüngeres „Ich“ Catull auswendig lernen wollte, um Erfolge beim anderen Geschlecht zu erzielen. In der Adoleszenz interessieren also die erotischen Sujets, im Alter hingegen der Abschiedsschmerz der Liebenden (in latyn' fehlen Indizien für eine Retrospektive, daher setzt man das Alter des Sprechenden mit dem des realen Autors gleich). Mit den literarischen Präferenzen vor der Pubertät setzt sich domašnee čtenie [Hauslektüre] (Cvetkov 2010a, 160) auseinander.

Der Titel (,Hauslektüre' ist eine Komponente des schulischen Literaturunterrichts), die zeitliche Angabe „beinahe in der kindheit“ (počti v mladenčestve) am Anfang von V. 1 sowie das beschriebene heimliche Lesen unter der Bettdecke charakterisieren den Protagonisten als Schulkind. Anders als in latyn' oder "čut' gormonal'naja nakryla grust'... “ fehlt im Text das grammatische „ich“. Der um einiges ältere Sprecher geht stärker 
auf Distanz, verwendet die 3. Pers. oder unpersönliche Formulierungen. In V.2 findet sich ein Verb in der 2. Pers. Sing. ohne Personalpronomen („listaeš iliadu“ [du blätterst in der ilias]). Es handelt sich um einen sogenannten allgemein-persönlichen Satz, der neben allgemeinen, sprichwortartigen Aussagen auch die wiederholte Handlung einer bestimmten Person bezeichnen kann, in der Regel des Sprechenden. Das Gedicht erzählt also von der eigenen Kindheitslektüre, ohne den Ich-Bezug grammatisch explizit zu machen, und mit der Möglichkeit einer Verallgemeinerung auf jedermann und beide Geschlechter.

Die Interessen des Kindes, das in domašnee čtenie unter der Bettdecke liest, richten sich auf die großen Kriegsmythen der griechischen Antike: Trojanischer Krieg (Str. 1), Perserkriege (Str. 2), die Eroberungen Alexanders der Große (Str. 3). Dass im Jahr 2008, aus dem die Gedichte im Band skazka na noč [märchen für die nacht] stammen (Cvetkov 2010a, 4), diese Sujets in dieser Kombination für den Autor aktuell geworden sind, hängt vielleicht damit zusammen, dass in den vorangehenden Jahren Hollywood-Verfilmungen eben dieser brachialen Legenden in die Kinos kamen: Troja (2004), Alexander (2004) und v. a. 300 (2007). ${ }^{9}$ Cvetkovs Junge bleibt allerdings im Bereich der literarischen Originale; bei der Interpretation als jüngeres Ich des Autors wäre die Lektüre zeitlich in den 1950er Jahren zu lokalisieren. Was interessiert den Jungen an den genannten Texten? In der Ilias ist es neben dem Kämpfen die mythologisch-märchenhafte Dimension, d. h. die reale Präsenz der Götter und durch menschliche Leidenschaften (nicht durch realpolitische Interessen) motivierte Krieg (V.4-6):

там близок зевс там полыхает грозно
копейная гекзаметрами бронза
и из-за баб ревнивая возня
„dort ist zeus nah dort flammt drohend
mit hexametern die speerbronze
und eifersüchtiges gezerre wegen der weiber“

Str. 2 thematisiert den zweiten Kriegsmythos, der den Jungen in seinen Bann schlägt, nämlich das Ausharren der Spartaner unter Leonidas an den Thermopylen bis zum letzten Mann. Während in Str. 1 die Beschreibung der die Kriegshandlungen auslösenden Motive als "Gezerre wegen der Weiber" ansatzweise eine Relativierung der kindlichen Begeisterung beinhalten, sind die Überschreibungen beim Thermopylen-Mythos stärker. Das gelesene Buch handelte laut V.9-12:

о несусветных подвигах войны
о мертвом камне выбранном посланцем
ступай мол друг и передай спартанцам
что вымерли не персы а они
„von den unglaublichen taten des krieges
von dem zum boten erwählten toten stein
freund los geh und richte den spartanern aus
dass nicht die perser ausgestorben seien sondern sie“

9 Zum Beitrag des Films an der Prägung (populärer) Bilder der Antike siehe Hardwick 2003, 7185 sowie zu Spartacus 37-43. Zumindest in den Überblicksdarstellungen zur Antikerezeption in Russland bleiben die neuen Medien oder auch literarisch-bildkünstlerische Mischformen wie Comic oder graphic novel bislang unberücksichtigt. 
Cvetkovs (idealer) junger Leser liest hier offenbar Herodots Historien (auch bei der Ilias handelt es sich eindeutig um keine Adaption à la Gustav Schwab, denn es werden Hexameter erwähnt). V.11f. paraphrasiert die in den Historien $(7,228)$ überlieferte Inschrift, die an die gefallenen Spartaner erinnert. Dabei verändert das Gedicht nicht nur das rhetorische Register - von erhaben-feierlich zu familiärer Umgangssprache ${ }^{10}{ }_{-}$, sondern auch die zu verkündende Botschaft. Zum einen steht anstatt des zu erwartenden $u$-merli (,sind gestorben“) vy-merli („sind ausgestorben“), was sich auf die Spartaner als Ethnos beziehen muss. Damit wird der Sinn des Ausharrens bis zum letzten Mann aus der Langzeitperspektive in Frage gestellt, denn überlebt haben offenbar die Perser. (In der Tat gehörte die Zukunft den Imperien.)

Zum anderen tilgt das Gedicht den ursprünglichen Grund, warum die Tat der Spartaner der Erinnerung wert sein soll. Laut Winkler (DNP VIII, 610) haben sie bei Herodot das 'Wort, den Befehl' (to rhēma) erfüllt, Lykurg $(1,109)$ machte daraus 'Sitte, Gesetz' (to nomimon). Diese Version bestimmte die spätere Rezeption, die die Thermopylen als Symbol für die Verteidigung der jeweils eigenen Kultur verstand (DNP VIII, 610, 613-618). In der russischen Herodotübersetzung von Georgij A. Stratanovskij (1901-1986) heißt es, die Spartaner seien „das Vermächtnis ${ }^{11}$ behütend“ gestorben (vgl. FN 9). Tief ins deutsche kulturelle Gedächtnis hat sich folgende, auf Schillers Der Spaziergang (1795) zurückgehende, Version eingeprägt:

„Wanderer, kommst du nach Sparta, verkündige dorten, du habest

Uns hier liegen gesehn wie das Gesetz es befahl.“

Im deutschen Kontext - Cvetkov lebte und arbeitete einige Jahre in München und verfügt über gute bis sehr gute Deutschkenntnisse (Cvetkov, Skvorcov 2007, 246: er lese Rilke im Original) - assoziiert man heute aber weniger Schiller als Heinrich Bölls Erzählung Wanderer, kommst du nach Spa... (publ. 1950), die das Epigramm in den Kontext des „totalen Krieges" einordnet (zu Böll: DNP Suppl. VIII, 617). Hinter Böll steht eine Rede Hermann Görings vom 30.01 .1943 (vgl. ebd.; Transkription der Radioübertragung: Krüger 2003, 387-401), die zum Durchhalten à la Leonidas im aktuellen Kampfe um „Freiheit oder Vernichtung" (398) an der Ostfront aufpeitschte:

„Und es wird auch einmal heißen: kommst du nach Deutschland, so berichte, du habest uns in Stalingrad liegen sehen, wie das Gesetz, das heißt, das Gesetz der Sicherheit unseres Volkes, es befohlen hat." (Krüger 1998, 397)

Diese Zuspitzung auf den Überlebenskampf findet sich in Cvetkovs Paraphrase, die die Interpretation des 20. Jahrhundert auf den Punkt bringt, allerdings die Rolle von Siegern und Verlierern tauscht.

Bei Cvetkov geht die kritische Überschreibung in Str. 3 weiter. Neben dem Alexander-Mythos werden Erzählungen von Helden und Führern generell als Lüge abgewertet (V.13-15):

10 Vgl. mit dem feierlichen Ton der Übersetzung von G. A. Stratanovskij: „Путник, пойди возвести нашим гражданам в Лакедемоне, / Что, их заветы блюдя, здесь мы костьми полегли.“

${ }^{11}$ Russ. zavet findet sich in den Begriffen für Altes und Neues Testament, bezeichnet also einen sakralen und eminent wichtigen Teil der eigenen Kultur. 
так сладко в юности читать неправду

что дескать топал александр к евфрату

все ерунда герои и вожди

„es ist so süß in der Jugend die unwahrheit zu lesen

dass alexander zum euphrat gestapft sei

alles quatsch die helden und die führer"

Der einzige Inhalt, der in der zweiten Hälfte der Strophe, die vom Altern handelt, von dem kritischen Urteil ausgenommen wird, ist Elena (V.17f.). Gemeint ist die Helena der Ilias und zugleich eine reale Elena aus dem nordrussischen Čerdyn'. Wie in latyn' halten also die Liebessujets stand, da sie an eigene Erinnerungen an die Liebe anknüpfen können. Für die Zweikämpfe der antiken Helden gilt dies nicht mehr, zu sehr unterscheiden sie sich von den realen Erfahrungen in den Schlachten der Moderne.

Trotz der Kritik an der naiven Begeisterung für militärische Abenteuer endet Strophe vier mit einer positiveren Gesamtwertung der antiken Texte. Im Alter, in dem das Leben seicht und ereignislos geworden sei, würden sie vor der geistigen Kapitulation retten (V.21-24):

жизнь к старости на случаи скупа

давно бы скис и уступил погоне

когда бы не елена в илионе

не яблоко не ксеркс и не судьба

„zum alter hin geizt das leben mit ereignissen

schon lange hätte [ich] schlapp gemacht und der verfolgung nachgegeben

wenn da nicht helena in ilion wäre

nicht der apfel nicht xerxes und nicht das schicksal“

\section{Kallisthenes und der Opportunismus der (Zeit-)geschichte: rasskaz očevidca}

Kritisch mit legendären Kriegern beschäftigt sich auch das letzte hier behandelte Gedicht rasskaz očevidca [erzählung eines augenzeugen], das im Band skazka na noč (Cvetkov 2010a, 121f.) dem schon erwähnten domašnee čtenie (Cvetkov 2010a, 160) vorausgeht. Im Zentrum stehen Lüge und Wahrheit des Alexander-Mythos, der im letzten Gedicht nur kurz angeschnitten wurde.

Der Titel „erzählung eines augenzeugen“ verweist auf die Spannung zwischen Realität und historischer Überlieferung. Auch oder gerade Berichte von Beteiligten sind bekanntlich bedingt vertrauenswürdig, da sie stark von persönlichen Interessen geprägt sind und/oder propagandistischen Zwecken dienen. Der Augenzeuge, der in Cvetkovs Gedicht in der 1. Pers. Sing. spricht, trägt keinen Namen. Die in Abschnitt 1 (V. 1-14) verstreuten Indizien ermöglichen jedoch die Verortung in der Zeit Alexanders des Großen (356-323):

на солнечных часах четвертый век
до нашей эры мобилизовали
большое войско и вперед на персов
я состою историком в строю
описывая ход событий скоро

Philologia Classica. 2018. Vol 13. Fasc. 1 


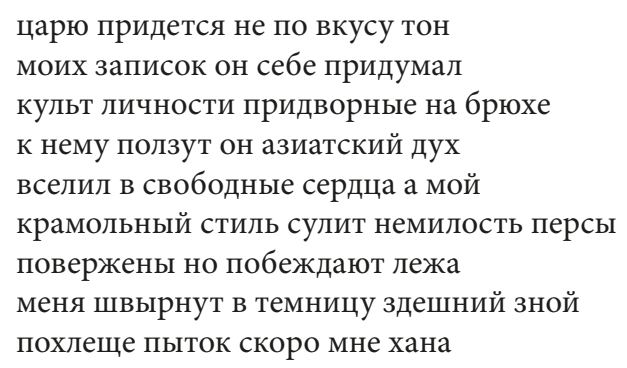

„auf der sonnenuhr ist es das vierte jahrhundert / vor unserer zeitrechung mobilisiert wurde / ein großes heer und vorwärts auf die perser / als historiker steh ich in reih und glied / beschreibe den lauf der ereignisse bald / wird dem zaren der ton meiner / aufzeichnungen nicht gefallen er hat sich einen / personenkult ausgedacht die höflinge kriechen / auf dem bauch zu ihm hin asiatischen geist hat er / in die freien herzen gegossen und mein / aufrührerischer stil birgt missgunst die perser / sind niedergerungen aber siegen auf dem boden liegend / man wirft mich in den kerker die hiesige glut / peitscht schlimmer als folter bald ist's aus mit mir"

Rasskaz očevidca ist ein Rollengedicht, es erzählt ein Geschichtsschreiber, was die veränderte, prosaischere Gedichtform erklärt: Die Verse folgen erneut einem syllabotonischen Bauprinzip (5-hebiger Jambus), aber es fehlen die Reime sowie die Einteilung in homogene Strophen; Enjambements verunklaren die Versgrenzen. Bei dem Chronisten, der im Auftrag Alexanders eine schmeichelhafte Darstellung von dessen Erfolgen anfertigen soll, Kritik an der Proskynese übt und in Ungnade fällt, handelt es sich um eine reale Person. Kallisthenes von Olynth begleitete Alexander auf den Feldzug gegen die Perser und verfasste eine Darstellung der "Taten Alexanders“ (dazu: Gilhaus 2017, 30-37). Das Werk überdauerte die Jahrhunderte nicht, erhalten sind Fragmente. Woher genau der polyglotte Cvetkov sein Wissen schöpfte, lässt sich in den meisten Fällen nicht genau bestimmen, da die Bezüge zu allgemein sind. Es sind keine konkreten Referenzen aus Kallisthenes' Alexandergeschichte verarbeitet.

In rasskaz očevidca geht es auch nicht primär darum, intensives Quellenstudium in Gedichtform zu gießen, sondern um eine breitere historische Perspektive, die auf eine Analogie zuläuft. So blickt Kallisthenes in Abschnitt 2 in einer Art Delirium in die Zukunft. Die ersten Stationen der Zeitreise stammen aus der englischen Geschichte: Wilhelm der Eroberer (1027/28-1087), der die Insel normannischer Herrschaft unterwarf, und Alfred der Große (848/849-899), der die angelsächsischen Königreiche vereinigte. Die Erwähnung von zwei Hälften eines Imperiums verweisen wohl auf Rom und Byzanz. Danach erreicht Kallisthenes seine Gegenwart - Darius flieht nach der Niederlage bei Gaugamela - und vollzieht einen Schritt in die Vergangenheit als „der große eroberer noch in pelle auf dem töpfchen brabbelt“ („poka velikij / zavoevatel’ v pelle na gorške / agukaet", V.24f.). Danach wechselt das Gedicht zurück zur weniger amüsanten Gegenwart, in der sich der Eingekerkerte in einem bewussten Anachronismus eine Schmerztablette wünscht, um die Folgen der Haftbedingungen zu mindern.

Am Ende des zweiten Abschnitts verkündet der Sprecher seinen Tod: „ja molča mertv“ (V.28) [ich bin schweigend tod], zu Beginn des dritten äußert er allerdings den Wunsch, man möge an sein Schicksal erinnern. Es klingt erneut das Thermopylen-Epigramm an: 
о путник передай

когда вернешься в грецию народу

вернее деду или лучше дяде

внучатому он академик минц

„oh wanderer teile dem volk

mit wenn du nach griechenland zurückkommst

oder eher dem großvater oder dem großonkel

er ist das akademiemitglied minc"

Von großer Relevanz für den Text ist der Adressat: „Volk“ (narod) wird verbessert zu Großvater und zu Großonkel. ${ }^{12}$ Zum einen soll also Verwandten die Todesnachricht zugestellt werden, was den Heroismus-Patriotismus des Originals aufhebt. Kallisthenes' Widerstand gegen die Tyrannis führt in den Tod, ohne irgendetwas zu bewirken. Zum anderen ist die Erwähnung des Onkels ausschlaggebend für die sich anschließende Übertragung der antiken Episode auf Ereignisse des 20. Jahrhunderts. Kallisthenes war mit Aristoteles verwandt und mit diesem eng verbunden. Anders als der Neffe vermied der Onkel die Kollision mit Alexander und verhielt sich damit opportunistischer. ${ }^{13}$

Welche Parallele zur russischen Geschichte in Cvetkovs Gedicht gezogen wird, hat schon der Begriff „Personenkult“ (kult ličnosti) in V.8, der sofort mit Stalin assoziiert wird, angedeutet. Der in V.32 erwähnte Name des „Mitglieds der Akademie der Wissenschaften" (akademik) Minc bestätigt diese Interpretation. Isaak I. Minc (1896-1991) war ein prominenter sowjetischer Historiker mit den Arbeitsschwerpunkten Bürgerkrieg und "Großer Vaterländischer Krieg" - modernen Entsprechungen der Perserkriege. Der Star der stalinistischen Historiographie fiel 1949 in Ungnade, überstand die Kampagne gegen ihn relativ unbeschadet, auf eine Professur an der Pädagogischer Hochschule degradiert, und stieg am Ende der 1950er erneut in Spitzenpositionen auf. Er gilt als prominenter typischer Vertreter des auf Anpassung bedachten Historikers der Sowjetzeit (siehe die Erinnerungen von Poljakov 2004, Ioffe 2004). Cvetkovs Allegorie beschreibt in antikem Gewand die 1930-40er Jahre, in denen auch loyale Opportunisten nicht vor Verdächtigungen eines paranoiden Autokraten sicher waren. ${ }^{14}$ Als Opportunist hatte der Alexanderbiograph Kallisthenes begonnen, und als solchen kann man Aristoteles einordnen, der dem Treiben seines früheren Schülers keinen Widerstand entgegensetzte. Da bei Cvet-

12 Das in Verbindung mit djadja kaum gebräuchliche vnučatyj weist auf eine entferntere Verwandtschaftsbeziehung hin (vnučatyj plemjannik wäre der Enkel von Bruder oder Schwester). Von Aristoteles als „Großonkel“ spricht Gilhaus $(2017,30)$.

${ }^{13}$ Ich danke der Redaktion und A. K. Gavrilov herzlich dafür, dass Sie mich noch einmal auf diese Verwandtschaftsbeziehung und v. a. auf Plutarch als intertextuellen Bezugspunkt hingewiesen haben.

${ }^{14}$ In Plutarchs Alexandervita (\$55) findet sich auch eine überraschende Parallele zu Stalins Verfolgungswahn: Alexanders Wut hätten sich auch gegen den Onkel, an dessen Loyalität der Herrscher ohne konkrete Veranlassung zweifelte, bzw. eine vermutete Verschwörergruppe gerichtet. Aufgrund bescheidener Griechischkenntnisse (Cvetkov, Skvorcov 2007, 245) dürfte Cvetkov auf eine Übersetzung zurückgegriffen haben. In der klassischen Übertragung ins Russische lautet das Zitat wie folgt: Alexander habe in einem Brief geschrieben: „ «[...] а софиста [d. h. Kallisthenes; M. R.] я еще накажу, как, впрочем, и тех, кто его прислал и кто радушно принимает в своих городах заговорщиков, посягающих на мою жизнь». Здесь Александр явно намекает на Аристотеля [...].“ (Plutarch 1963, 436). Auf Deutsch: „„,[...] den Sophisten [d. h. Kallisthenes; M. R.] aber werde ich selbst bestrafen und ebenso, die ihn ausgesandt haben, und diejenigen, welche Leute, die Böses gegen mich im Schilde führen, in ihren Städten aufnehmen“, womit er ganz offen auf Aristoteles zielt [...]." (Plutarch 1994, 147/149) 
kov Interpunktionszeichen fehlen, kann man die Äußerung „er ist das akademiemitglied minc" dabei sowohl als Kommentar des Sprechers lesen, der Aristoteles mit Minc gleichsetzt, als auch (so der treffende Vorschlag von D. Keyer) als wörtliche Rede des auf seinen Tod wartenden Neffen, der dem Onkel dessen Mitläufertum vorwirft (vielleicht sogar als „er sei ein akademik minc“ im Sinne eines Schimpfworts).

Auch das Motto, das eine gesonderte Erwähnung verdient, verweist auf die 1940er Jahre. Cvetkov zitiert den Anfang eines Gedichts von Zbigniew Herbert (1924-1998), einem der wichtigsten polnischsprachigen Dichter der 2. Hälfte des 20. Jahrhunderts. Das Zitat aus Herberts Raport z oblężonego miasta [Bericht aus einer belagerten Stadt] (1982, publ. 1983) steht im polnischen Original und ohne Übersetzung, so dass das Motto nur von Leserinnen und Lesern verstanden werden kann, die mit Sprache und Kultur vertraut sind. ${ }^{15}$ Auch bei Herbert spricht eine imaginäre Figur, ein zum Chronisten bestimmter anonymer Alter. Er erzählt von einer Belagerung, die einer konkreten Zuordnung entzogen ist (antike Realien vermischen sich mit solchen aus anderen Zeiten). Im polnischen Kontext denkt man sofort an die Zerstörung Warschaus im Jahr 1944 durch die deutsche Wehrmacht, die im erbitterten Kampf Haus um Haus einnahm. Dies wäre ein modernes Analogon zum Thermopylen-Mythos. Die spätere Anpassung gewisser Fakten durch die Historiker der Sowjetunion und der Volksrepublik Polen an die ideologisch-propagandistischen Erfordernisse entspräche der Situation von Kallisthenes sowie Minc, die historische Realität an die Wünsche Ihrer Auftraggeber anpassen zu müssen.

Die Antike liefert gerade in rasskaz očevidca kein Ideal, das einer schlechteren Gegenwart zum Vorbild dienen könnte. Sie liefert eher ein (vertrautes) Muster, das sich in der Geschichte wiederholt und auch in der jüngeren Vergangenheit zu finden ist. Einige Momente verweisen konkret auf die Stalinzeit, das Gedicht hält aber zugleich, wie Herberts Bericht aus einer belagerten Stadt, eine allgemein-allegorische Perspektive offen - vielleicht, um selbst in Hunderten von Jahren noch verständlich und aktuell zu sein. Neben Erfahrungen von Liebe und Abschied gehört wohl auch das Wissen um die von der Geschichtsschreibung geforderten und von ihr beförderten Propagandalügen zu den ewigen Themen.

\section{Literatur*}

Cvetkov A. Sbornik pes dlia zhizni solo [a collection of pieces for solo life]. Ann Arbor, Ardis, 1978 (in Russian) Cvetkov A. Sostoianie sna [a state of sleep]. Ann Arbor, Ardis, 1981. (in Russian)

Cvetkov A. Edem [Eden]. Ann Arbor, Ardis, 1985. (in Russian)

Cvetkov A. Divno molvit' [wonderful to utter]. St. Petersburg, Pushkinskii fond, 2001. (in Russian)

Cvetkov A. Prosto golos [just a voice]. Moscow, Nezavisimaia gazeta, 2002. (in Russian)

Cvetkov A. Shekspir otdykhaet [Shakespeare at rest]. St. Petersburg, Pushkinskii fond, 2006. (in Russian)

Cvetkov A. Latyn' [latin]. Znamja 2007a, 1, 94-99. (in Russian)

Cvetkov A. Imena liubvi [names of love]. Moscow, Novoe izdatel'stvo, 2007b. (in Russian)

Cvetkov A. Rovnyi veter [the even wind]. Moscow, Novoe izdatel'stvo, 2008. (in Russian)

Cvetkov A. Skazka na noch' [bedtime story]. Moscow, Novoe izdatel'stvo, 2010a. (in Russian)

Cvetkov A. Detektor smysla [sense detector]. Moscow, ARGO-RISK - Knizhnoe obozrenie, 2010b. (in Russian)

Cvetkov A. Ontologicheskie napevy [ontological tunes]. New York, Ailuros, 2012. (in Russian)

http://www.elenasuntsova.com/tsvetkov (06.03.2018).

${ }^{15}$ Cvetkovs Interesse an polnisch-russischer Geschichte und Kenntnisse der polnischen Sprache zeigt auch das Gedicht katyń (Cvetkov 2010b, 127), das Russisch und Polnisch mischt.

* Übersetzung ins Englische von der Red. vorgenommen. 
Cvetkov A. Salva veritate. New York, Ailuros, 2013, http://www.elenasuntsova.com/tsvetkov_salva_veritate (06.03.2018). (in Russian)

Cvetkov A. Vse eto, ili Eto vse. Sobranie stikhotvorenii v dvukh tomakh [all that or is that all. A Collection of Poems in Two Volumes]. New York, Ailuros, 2015, http://www.elenasuntsova.com/tsvetkov_all_of_it (06.03.2018). (in Russian)

Cvetkov A. Iz raznykh avtorov i iazykov [from various authors]. Inostrannaia literatura 2017, 5, http://magazines.russ.ru/inostran/2017/5/iz-raznyh-avtorov-i-yazykov.html (04.03.2018). (in Russian)

Cvetkov A., Skvorcov, A. „Nado ne gordit'sia, a znat'...“ [“One must not be proud of, but know] [Interview]. Voprosy literatury 2007, 3, 239-251. (in Russian)

Chagi S. K. K voprosu o poeticheskoi distopii: „Post aetatem nostram” Iosifa Brodskogo [On Poetic Dystopia: Post aetatem nostram by Iosif Brodskii], in: O. I. Glazunova (ed.) Iosif Brodskii v XXI veke (I. Brodskii in 20th century). St. Petersburg, Filologicheskii fakul'tet SPGU, 2010, 177-181. (in Russian)

Cypilëva P. A. Retseptsiia antichnoi kul'tury v tvorcheskom soznanii Ars. Tarkovskogo, A. Kushnera, S. Stratanovskogo [The Reception of Ancient Culture in Creative Consciousness of Ars. Tarkovskii, A. Kushner, S. Stratanovskii] [avtoreferat]. Tomsk, 2016. (in Russian)

Frajlich A. The Legacy of Ancient Rome in the Russian Silver Age. Amsterdam - New York, Rodopi, 2007.

Gilhaus L. (Übers., Komm.). Fragmente der Historiker: Die Alexanderhistoriker [FGrHist 117-153]. Stuttgart, Anton Hirsemann, 2017.

Golodnikov L. V.(sost.). Antichnost'v russkoi poèzii (XVIII-nachalo XX veka) [Antiquity In Russian Poetry (XVIII-early XX cent.)]. St. Petersburg, IC „Gumanitarnaia Akademiia”, 2012 (in Russian).

Gresta E. Il poeta è la folla. Quattro autori moscoviti: Vsevolod Nekrasov, Lev Rubinštejn, Michail Ajzenberg, Aleksej Cvetkov. Bologna, CLUEB, 2007.

Gvozdeva T. B. (sost.). Mir antichnosti v zerkale russkoi poezii: antologiia. T. 1: mifologiia [The World of Antiquity in the Mirror of Russian Poetry]. Moscow, Literaturny institut im. A. M. Gor'kogo, 2014. (in Russian).

Hardwick L. Reception Studies. Oxford, Oxford UP, 2003.

Ioffe G.Z. Iz vospominanii ob akademike I. I. Mintse: „Ostal'noe vam dast sovetskaia vlast“" [From the Memories of the Academician Minc. The Soviet Power uill Supply The Rest.]. Otechestvennaia istoriia, 2004, 4, 152-158. (in Russian)

Knabe G.S. (ed.). Antichnoe nasledie v kul'ture Rossii [Ancient Heritage In Russian Culture]. Moscow, RNII kul'turnogo i prirodnogo naslediia, 1996. (in Russian)

Krüger P. Etzels Halle und Stalingrad. Die Rede Görings vom 30.1.1943. Die Nibelungen. Sage - Epos Mythos. Hg. von J. Heinzle, K. Klein, U. Obhof. Wiesbaden, Reichert, 2003. 375-403.

Levitsky A. Tsvetkov Aleksei. Handbook of Russian Literature. Ed. by V. Terras. New Haven — London, Yale UP, 1985, 487.

Mal'chukova T.G., Dechtjarenok A. B., Nilova A. Ju. (eds). Klassitsizm i neoklassitsizm v russkoi literature XVIII-XIX vv. Petrozavodsk, Izd. PetrGU, 2011. (in Russian)

Martirosova Torlone Z. Russia and the Classics: Poetry's Foreign Muse. London, Duckworth, 2009.

Möllendorff P.von, Simonis A., Simonis L. (Hg.) Historische Gestalten der Antike. Rezeption in Literatur, Kunst und Musik. Stuttgart - Weimar, J. B. Metzler, 2013.

Poljakov Ju. A. Iz vospominanii ob akademike I. I. Mintse: rovesnik epokhi [From the Memories of the Academician Minc: Coeval the epoch]. Otechestvennaia istoriia 2004, 4, 144-152.

Plutarch. Sravnitel'nye zhizneopisaniia. V trekh tomakh. Tom 2. Izd. Podgotovili M.E. Grabar'-Passek i S.P.Markiš. Moskva, Izd-vo AN SSR, 1963, http://publ.lib.ru/ARCHIVES/P/PLUTARH/_Plutarh. html 002 (19.04.2018). (in Russian)

Plutarch. Fünf Doppelbiographien. Griechisch und Deutsch. Übers. von K. Ziegler und W. Wuhrmann, ausgew. von M. Fuhrmann. Bd. 1. Darmstadt, WBG, 1994.

Riedel V. Antikerezeption in der deutschen Literatur vom Renaissance-Humanismus bis zur Gegenwart. Eine Einführung. Stuttgart - Weimar, J. B. Metzler, 2000.

Smith G. S. Aleksej Cvetkov's Lost Paradise. Slavic and East European Journal 1986, 30/4, 541-552.

Seidensticker B., Habermehl P. Unterm Sternbild des Hercules. Antikes in der Lyrik der Gegenwart. Frankfurt a. M. - Leipzig, Insel, 1996.

Skvorcov A. Ė. Vospriiatie antichnosti v poezii Iosifa Brodskogo i Alekseia Tsvetkova [The Reception of Antiquity in the Poetry of Iosif Brodskij and Alexei Cvetkov]. Russkaia sopostavitel'naia filologiia: sostoianie i perspektivy. Ed. by K. R. Galiullina. Kazan', Izd. Kazan. Universiteta, 2004, 324-326. (in Russian) 
Skvorcov A.Ė. Nesvoevremennaia sovremennost (O poezii Alekseia Tsvetkova) [Untimely Modernity (On the Poetry of Alexei Cvetkov)]. Znamja 2008, 8, 189-193. (in Russian)

Skvorcov A.Ė. Retseptsiia i transformatsiia poeticheskoi traditsii v tvorchestve O. Chukhontseva, A. Cvetkova i S. Gandlevskogo [avtoreferat]. [Reception and Transformation of Poetic Tradition in the Work of O. Čuchoncev, A. Cvetkov and S. Gandlevskii]. Kazan', 2011. (in Russian)

Tacho-Godi A. A. et al. (eds). Muzy u zerkala: antichnye motivy v russkoi literature [The Muses and the Mirrors: ancient motifs In Russian Literature]. Moscow, Novyi chronograf, 2015. (in Russian)

Uspenskaja A. V. Antichnost' i russkaia literatura: motivy, obrazy, idei [Antiquity and Russian Literature: Motifs, Images, Ideas]. St. Petersburg: Izd. SPbGUP, 2008. (in Russian)

Walde Ch., Egger B. (Hg.) Die Rezeption der antiken Literatur. Kulturhistorisches Werklexikon. Stuttgart Weimar, J. B. Metzler, 2010.

Wes M. A. Classics in Russia. 1700-1855. Between Two Bronze Horsemen. Leiden - New York - Köln, E. J. Brill, 1992.

\section{Об универсальности любви и о вечных неправдах истории. Рецепция античности в поэзии Алексея Цветкова}

\section{Марион Рути,}

Обозрения имеющихся исследований о рецепции античности в русской литературе обнаруживают пространства, мало или вовсе не затронутые наукой. Вне поля зрения остается, например, почти вся постсоветская литература. Между тем, античность для современной русской поэзии остается более чем актуальной и по-новому проблематизируется. Статья рассматривает в вышеозначенной перспективе (отражения античности на современном культурном и историческом фоне) творчество известного поэта советского андеграунда и русского зарубежья Алексея Цветкова ( $\left.{ }^{\star} 1947\right)$, ставшего после более чем десятилетнего молчания и возвращения в большую литературу в 20012006 гг. одним из важнейших представителей современной русской поэзии. Античные темы в поэзии Цветкова центральны. Около двадцати его текстов концентрируются на античности. В статье подробно анализируются три стихотворения: «латыны», «домашнее чтение» и "рассказ очевидияа». В них, как и в других своих произведениях, фокусирующихся на античных - будь то мифических или исторических - сюжетах, Цветков размышляет о возможности проникновения классической древности в современность и жизнеспособности античной литературы. Подобные темы можно было бы назвать затертыми, однако автор умеет по-своему концептуализировать известный материал и вписать его в контекст современности. Разбираемые в статье стихи заострены на спорности традиционного восприятия известнейших античных сюжетов. Разрабатываются проблематичные с сегодняшней точки зрения - учитывая громадность традиции - темы: Троя, Фермопилы, империалистический проект Александра. Цветков связывает участь Каллисфена, историографа Александра, с жертвами сталинизма и (с отсылкой к польской литературе) с трагедией Варшавского восстания.

Ключевые слова: Алексей Цветков, античное наследие в современной русской поэзии. 